\title{
Teste Estrutural de Integração de Programas de Aplicação de Banco de Dados Relacional
}

\author{
Edmundo Sérgio Spoto ${ }^{1}$, Plínio de Sá Leitão Junior ${ }^{2}$, Mario Jino ${ }^{2}$ e \\ José Carlos Maldonado ${ }^{3}$ \\ ${ }^{1}$ UNIVEM/FEESR - Av. Hygino Muzzi Filho, 529 - Cmps Univ. Cep: 17.525-901, \\ Cx. Postal 2041, Marília - SP, Brasil - (dino@fundanet.br ) \\ ${ }^{2}$ DCA/FEEC/UNICAMP - Av. Albert Einstein, 400, Cep: 13083-970, Cx. Postal 6101, \\ Campinas - SP, Brasil (plinio@dca.fee.unicamp.br; jino@dca.fee.unicamp.br) \\ ${ }^{3}$ ICMC-USP - Av. Dr. Carlos Botelho, 1465, - Cep: 13560-970, Cx. Postal 668, \\ São Carlos - SP, Brasil (jcmaldon@icmc.usp.br)
}

\begin{abstract}
The search for quality in Relational Database Applications (RDA) involves the definition of testing approaches that consider persistent data flow. The structural testing technique is based on the internal structure of programs; it is applied in the unit testing of programs and in the integration testing of those units. This work explores the analysis of persistent dataflow in $R D A$, to determine requirements for structural integration testing. Specifically, an approach is proposed for the structural integration testing of programs with embedded SQL, where the persistence of data establishes data flow associations. The approach considers intra-modular and inter-modular integration testing to define test requirements. The testing criteria proposed are based on the call structure of units and on the exclusive data dependence, focused on the relationships of persistent dataflow.
\end{abstract}

Resumo. A busca pela qualidade de Aplicações de Banco de Dados Relacionais (ABDR) envolve a definição de abordagens de teste que considerem o fluxo de dados persistentes. A técnica de teste estrutural é baseada na estrutura interna de programas; ela é aplicada no teste de unidade de programas e na integração dessas unidades. Este trabalho explora a análise de fluxo de dados persistentes em ABDR, para determinar requisitos para teste estrutural de integração. Especificamente, uma abordagem é proposta para o teste estrutural de integração em programas com SQL embutida, onde a persistência de dados estabelece associações de fluxo de dados. A abordagem considera o teste de integração intra-modular e intermodular para definir os requisitos de teste. Os critérios de teste propostos estão baseados na estrutura de chamada entre unidades e na dependência exclusiva de dados, focados nas relações de fluxo de dados persistentes.

\section{Introdução}

A manipulação de dados persistentes desempenha um importante papel em soluções implementadas por software. A persistência de dados é um atributo ligado à necessidade por dados não voláteis na execução de aplicações. Aplicações que manipulam dados persistentes são distintas das aplicações convencionais, pois incorporam tais dados ao espaço de entrada e de saída durante a sua execução.

O uso de bancos de dados relacionais tem-se difundido em aplicações que manipulam dados persistentes. Tais aplicações, denominadas Aplicações de Banco de 
Dados Relacional (ABDR), tipicamente recuperam e ou atualizam as relações das bases de dados. Essas relações representam os dados persistentes que, em conjunto com as variáveis declaradas no programa, compõem as variáveis de uma aplicação.

Similares a programas convencionais, ABDRs requerem esforços para o controle e para a melhoria de sua qualidade. Teste de software é uma atividade usada para dar evidências da confiabilidade e da qualidade de software. Assim, é pertinente a busca por abordagens voltadas ao teste de ABDRs. Especificamente, a técnica de teste estrutural é baseada na estrutura interna de programas, focando o fluxo de controle e o fluxo de dados. O teste se divide em 2 estágios: teste de unidade de programa - menor parte executável de um programa, como uma função ou procedimento - e teste de integração dessas unidades. O foco deste artigo é a aplicação da técnica de teste estrutural no estágio de integração, no contexto de aplicações que manipulam bancos de dados relacionais.

A geração de bases de dados é uma área pertinente ao teste de aplicações que manipulam dados persistentes. A utilização de bases dedicadas à atividade de teste usualmente alcança uma reduzida amostra dos dados, a qual pode não ser representativa dos cenários reais de uso, especialmente em termos de teste de performance. Por outro lado, o uso de bases reais pode potencialmente expor dados protegidos contra acessos não autorizados. Wu et al. investigam técnicas para a aplicação de bases de produção ao teste de aplicações, sem revelar qualquer informação confidencial presente nessas bases [WU04]; a idéia é simular ambientes reais para propósitos de teste, mantendo a privacidade dos dados na base original. Chays et al. também estudam a geração de bases de teste, apresentando uma abordagem baseada na indicação do testador de dados típicos para cada atributo da base de dados [CHA00]; em [CHA03] é apresentada uma ferramenta que automatiza esta abordagem, auxiliando a aplicação de casos de teste e a checagem se as saídas e o novo estado da base de dados estão consistentes com o comportamento esperado.

Um aspecto pertinente é a determinação de requisitos que deveriam ser cumpridos durante o teste, visando a aumentar a propensão para revelar os defeitos existentes no software. Esses requisitos são comumente definidos por critérios de teste e estão associados a modelos. No caso do teste estrutural, o modelo de fluxo de controle é usado para definir critérios que exploram a transferência de fluxo de controle da aplicação. Para exemplificar, considere o grafo de fluxo de controle (GFC) de um programa $P$, que é um grafo dirigido, denotado por $G(P)=(N, A, e, s): N$ é o conjunto de nós, onde cada nó é uma seqüência de comandos executados sem transferência de controle, também denominado bloco; $A$ é o conjunto de arcos representando a transferência de controle entre nós; $e$ é o nó de entrada; e $s$ é o nó de saída [CLA89, MAL91]. O critério todos os nós requer a execução de todos os nós durante o teste; o critério todos os arcos requer o exercício de todas as transferências de controle.

Por outro lado, o critério todos os caminhos explora as noções de caminho e de caminho completo: um caminho é uma seqüência finita de nós $\left(n_{1}, n_{2}, \ldots, n_{k}\right), k \geq 2$, tal que, para todo nó $n_{i}, 1 \geq i \geq k-1$, existe um arco $\left(n_{i}, n_{i+1}\right)$ para $i=1,2, \ldots k-1$; um caminho completo é um caminho cujos nós inicial e final são, respectivamente, o nó de entrada e o nó de saída. O critério todos os caminhos requer que todos os caminhos completos sejam exercitados durante o teste; em geral, esse critério é impraticável, visto que a quantidade de caminhos completos é elevada, sobretudo quando existem laços. Os elementos exigidos por um critério de teste são denominados elementos requeridos.

Um outro modelo, denominado modelo de fluxo de dados, representa as ocorrências de definição e de uso de variáveis de programa. Uma definição de variável 
ocorre quando um valor for armazenado em uma posição de memória. Um uso de variável ocorre quando houver uma recuperação de valor de uma posição de memória associada à variável, sem que se esteja definindo a variável. Os critérios de teste baseados no fluxo de dados determinam requisitos de teste para estabelecer associações entre a definição e o uso de variáveis - associações def-uso; por exemplo, se uma variável é definida em um nó do programa, é necessário exercitar caminhos entre essa definição e (potenciais) usos dessa variável. É intuitivo que se um valor for atribuído a uma variável, esse valor deverá ser usado no programa. Tais critérios são em geral mais exigentes do que os critérios todos os nós e todos os arcos, pois demandam testes mais rigorosos, e menos exigentes do que o critério todos os caminhos. Os critérios propostos por Rapps e Weyuker exigem a ocorrência explícita de uso de variável nos elementos requeridos [RAP82, RAP85]; a proposta de Maldonado et al. não exigem a ocorrência explícita de uso de variável e usa a idéia de potencial uso [MAL88, MAL91]. Em ABDR, a análise de fluxo de dados deve contemplar as variáveis persistentes pois essas variáveis estabelecem associações adicionais às associações de programas convencionais. Critérios específicos foram propostos em [SPO00a], visando a exercitar o teste de ABDR para variáveis tabelas presentes nesses programas. As variáveis tabelas possuem um enfoque mais amplo por serem variáveis persistentes cuja definição só é concretizada quando for validada a transação da base de dados (utilizada pelo comando COMMIT) [SPO00b].

Explorar relações de fluxo de dados persistentes em ABDR envolve aspectos importantes como: definir um modelo de fluxo de controle que inclua os comandos de acesso à base de dados e às estruturas de tratamento de exceção típicas nessas aplicações; analisar as ocorrências de definição e de uso de dados persistentes; especificar como as relações de fluxo de dados são caracterizadas entre as unidades de programa e entre programas; definir um modelo que contemple as relações de fluxo de dados; estabelecer requisitos de teste com base nos modelos de fluxo de controle e de fluxo de dados.

O problema que norteia este trabalho é a carência de critérios de teste estrutural de integração para ABDR, envolvendo o uso da SQL (structured query language) para a manipulação de dados persistentes; programas com essa característica são chamados de programas com SQL embutida. Os critérios baseados em fluxo de dados para programas convencionais são inadequados para variáveis persistentes, pois estas possuem particularidades como: referência à mesma tupla para caracterizar uma associação def-uso, aspectos de consistência transacional que requerem a confirmação (commit) ou o cancelamento (rollback) de transações e o relacionamento entre as relações da base de dados. Leitão et al. discutem questões relativas a defeitos em comandos SQL que manipulam dados persistentes, estudam como falhas podem ser manifestadas e constroem um mapeamento entre tipo de defeitos e dimensões de falhas visando a abstrair os fatores que influenciam a propagação de defeitos até a saída de execução de comandos [LEI05].

O artigo explora o teste de integração, que é executado após o teste de unidades de programa, e representa uma técnica sistemática para construir a estrutura do software e para revelar defeitos associados à interação entre unidades. Requisitos para o teste de integração são estudados, baseados na análise de fluxo de dados persistentes. Basicamente, trabalha-se com a noção de módulo, que neste artigo representa um programa de aplicação. São sugeridos requisitos de teste de integração intra-modular e 
inter-modular, inspirados, respectivamente, em associações def-uso entre unidades de um programa e entre programas.

Os critérios de teste propostos baseiam-se em relações de fluxo de dados devido à dependência propriamente dita de dados persistentes ocasionadas nas definições que uma unidade $U_{A}$ ocasiona na tabela da base de dados e seu respectivo uso ocasionado por outra unidade $U_{B}$.

Introduzem-se na Seção 1 o contexto, o problema e a questão investigada no trabalho; na Seção 2, são estudados os aspectos de fluxo de controle e de fluxo de dados abstraídos para ABDRs com SQL embutida, onde se ressalta a dependência de dados devido à persistência de dados; o teste estrutural de integração para ABDRs, baseado na dependência de dados persistentes, é explorado na Seção 3; na Seção 4 introduzimos os critérios de teste de integração propostos, classificando-os como intra-modular e intermodular, e apresentamos um exemplo de seus ; as conclusões são apresentadas na Seção 5 .

\section{Definições Básicas e Terminologia}

O teste estrutural de programas de ABDR com SQL embutida possui características similares às do teste de programas convencionais. As técnicas de teste definidas para programas convencionais podem ser aplicadas em ABDRs a partir de algumas adaptações, tendo em vista a presença de comandos SQL, variáveis tabela e variáveis host, não existentes em programas convencionais. Os conceitos básicos apresentados nesta seção são os relativos aos critérios estruturais de teste de programas convencionais, modificados e estendidos para os critérios estruturais de teste utilizados em ABDR. Na elaboração da abordagem de teste foi necessário: i) adaptar os conceitos e critérios de testes de unidade e de integração de programas convencionais para programas de ABDR; e ii) criar novos critérios de teste estrutural específicos para o escopo de banco de dados relacional (BDR).

Uma aplicação de banco de dados relacional é composta por um ou mais Módulos; cada Módulo ${ }^{1}$ é composto por um conjunto de Unidades de Programa (UP), que são os procedimentos e funções do programa; um Módulo $\operatorname{Mod}_{i}$ pode ser

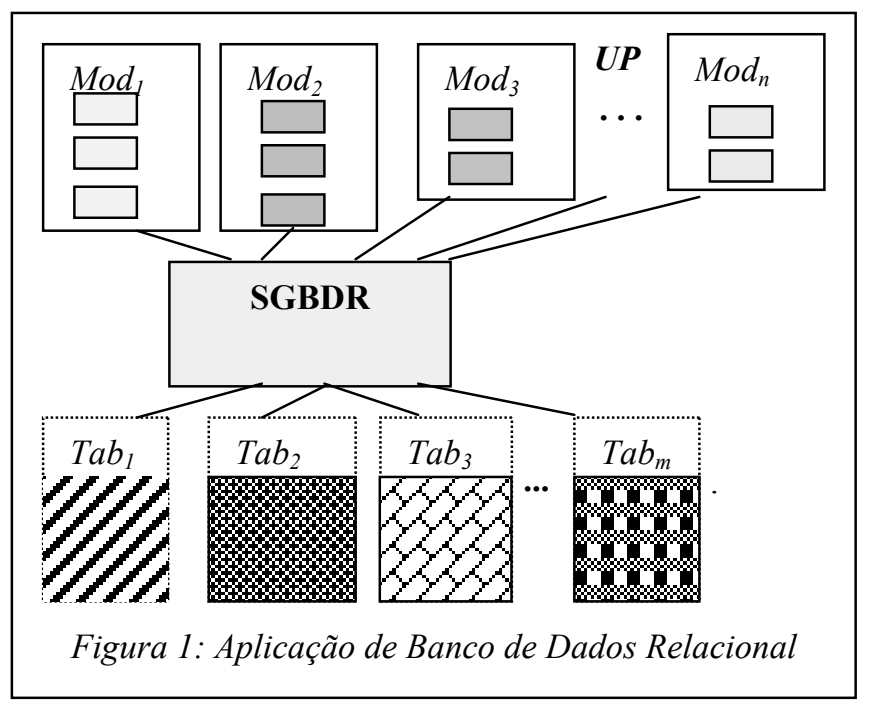
decomposto em várias $U P \mathrm{~s}$, onde 1 $\leq i \leq n$. Os Módulos geralmente podem ser codificados em diferentes linguagens, denominadas linguagens hospedeiras com SQL embutida. Cada UP é uma seqüência finita de comandos da linguagem hospedeira e comandos da linguagem SQL. A base de dados é representada pelas tabelas $T a b_{j}$ onde $1 \leq j \leq m$. A Figura 1 apresenta a idéia geral de uma ABDR composta por: módulos $\operatorname{Mod}_{1}, \operatorname{Mod}_{2}, . ., \operatorname{Mod}_{n}$, com suas respectivas unidades UPS, e as tabelas de $T a b_{1}, T a b_{2}, \ldots, T a b_{m}$.

Similarmente a programas convencionais, a estrutura de controle de programas de aplicação também pode ser representada por um grafo de fluxo de

\footnotetext{
${ }^{2}$ A palavra Módulo usada nesta abordagem corresponde a um programa de ABDR.
} 
controle. Entretanto, devido à existência dos comandos SQL, a representação gráfica de uma unidade de programa é um grafo de fluxo de controle estendido para ABDR com uso de SQL. A representação de grafo de programa acomoda os comandos executáveis da SQL em nós especiais, um em cada nó. Como notação gráfica, adotaram-se nós circulares para representar os blocos de comandos da linguagem hospedeira e de comandos declarativos da SQL, e nós retangulares para representar os comandos executáveis da SQL: INSERT, DELETE, UPDATE, SELECT, COMMIT, ROLLBACK, entre outros; um exemplo de GFC é exibido na Figura 2.

\subsection{Fluxo de Controle de Programa de ABDR}

O grafo de programa para representar uma UP de uma ABDR é denotado por G(UP) $=$ $\left(N^{B D}, E, n_{\text {in }}, n_{\text {out }}\right)$ onde: $N^{B D}=N_{h} \cup N_{S}, N_{h}$ é o conjunto de nós provenientes da

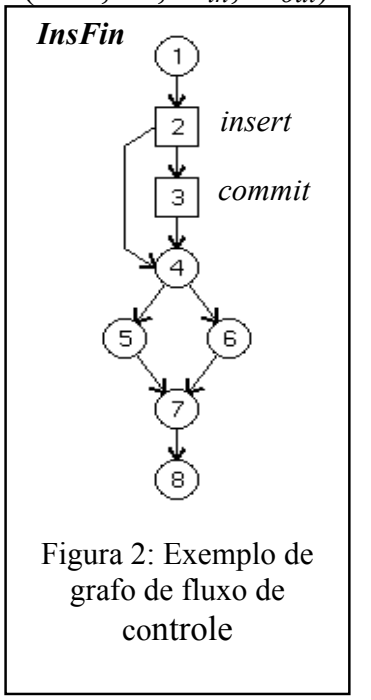
linguagem hospedeira e $N_{S}$ é o conjunto de nós provenientes da linguagem SQL; $E$ é o conjunto de arcos onde $E \subseteq N^{B D} \times N^{B D}$; o nó $n_{\text {in }} \in N_{h}$ é o nó de entrada e $n_{\text {out }} \in N^{B D}$ é o nó de saída do grafo de programa. $O$ grafo de programa estabelece uma correspondência entre os comandos do programa representados pelos conjuntos de nós $N_{h}$ e $N_{S}$, indicando os possíveis fluxos de controle entre os nós através dos arcos [SPO00b]. Os conjuntos $\operatorname{Arc}_{\text {in }}(i)$ e $\operatorname{Arc}_{\text {out }}(i)$ representam os conjuntos de $\operatorname{arcos}$ que, respectivamente, chegam e saem do nó $i$. A Figura 2 mostra o grafo de programa para a Unidade InsFin, que é uma unidade de programa extraída de [SPO00b], cuja linguagem hospedeira é a Linguagem $\mathrm{C}++$.

Para o tratamento de exceção foi utilizado o comando declarativo da SQL "EXEC SQL WHENEVER SQLERROR GOTO end"; no exemplo da Figura 2, este comando está no nó 1 (junto com os comandos da Linguagem $\mathrm{C}++$ ) e ocasiona a existência do arco $(2,4)$ com a condição de um desvio condicional para o rótulo "end" presente no nó 4 .

\subsection{Fluxo de Dados de Programa de ABDR}

A análise do fluxo de dados baseia-se nos tipos de ocorrência das variáveis de um programa de aplicação. Neste caso, podem existir três tipos de variáveis: i) variáveis de programa: são as variáveis manipuláveis somente por comandos da linguagem hospedeira; ii) variáveis host: são as variáveis que estabelecem os fluxos de dados entre a base de dados e o programa; e iii) variáveis tabela: são as variáveis tabela básicas (variáveis persistentes que compõem a base de dados) e as variáveis tabela de visão (tabelas virtuais derivadas das tabelas básicas e tratadas como variáveis locais ao Módulo de Programa). Cada tipo de variável tem o seu escopo de validade. Uma variável de programa é processada apenas no âmbito da linguagem hospedeira, uma variável host é processada tanto na linguagem hospedeira como na linguagem SQL e uma variável tabela é processada apenas na linguagem SQL e não precisa ser declarada no escopo do Módulo de Programa.

As variáveis host podem ser definidas e usadas em qualquer parte do programa (na linguagem hospedeira ou na linguagem SQL); denominamos de $s$-uso o uso de SQL relacionado às variáveis host e às variáveis tabelas que são usadas nos comandos da SQL. Para distinguir do uso na memória interna ocasionada pelas variáveis host, as 
variáveis tabela são definidas e usadas em memórias secundárias. Neste caso denominaremos de $t$-uso o uso da variável persistente ou variável tabela [SPO00b].

A abordagem adotada de teste estrutural para programas de ABDR considera dois tipos de fluxos de dados para as variáveis tabela: $i$ ) fluxo de dados intra-modular (aborda o teste de unidade e teste de integração intra-módular); e ii) fluxo de dados inter-modular; em ambos os casos, a análise de fluxo de dados é usada para abstrair requisitos para o teste de integração intra-modular e inter-modular. O fluxo de dados intra-modular é observado entre as UPS de um mesmo módulo; o fluxo de dados intermodular ocorre entre UPS de módulos distintos.

O teste de integração é aplicado depois que todas as unidades que compõem um programa foram devidamente testadas, isoladamente (teste de unidade). Para o teste de unidade em programas de aplicação, existe uma diversidade de critérios de teste estrutural, baseado em fluxo de controle e em fluxo de dados [RAP85, MAL91] que podem ser aplicados [SPO00a].

A dependência de dados é um aspecto fundamental associado à interação entre Unidades de Programas e Módulos da Aplicação. As dependências de dados existentes entre as UPS de programas convencionais são ocasionadas pelas variáveis globais ou por variáveis passadas por parâmetros por comandos de chamadas. ABDRs possuem dependências de dados baseadas nos comandos de chamada, como acontece nos programas convencionais e dependências de dados baseadas nas tabelas da base de dados. As dependências de dados entre as UPS e os Módulos de Aplicação de uma ABDR dão ensejo a novas abordagens de integração, propostas neste artigo.

Para apresentar os conceitos de teste de integração utilizados em programas de ABDR, foram apresentadas algumas definições importantes para o entendimento deste artigo. Um c-uso afeta diretamente uma computação que está sendo realizada, ou permite observar o valor de uma variável definida anteriormente. Um $p$-uso afeta diretamente o fluxo de controle do programa. Um caminho é simples se todos os nós que o compõem, exceto possivelmente o primeiro e o último, são distintos. Se todos os nós são distintos, diz-se que o caminho é um caminho livre de laço. Um caminho ( $i, n_{1}$, $\left.\ldots, n_{m}, j\right), m \geq 0$, com uma definição da variável $x$ no nó $i$ e que não contenha redefinição de $x$ nos nós $n_{1}, \ldots, n_{m}$ é chamado de caminho livre de definição com respeito a (c.r.a) $x$ do nó $i$ ao nó $j$ e do nó $i$ ao arco $\left(n_{m}, j\right)$. Um nó $n_{i}$ possui uma definição global de uma variável $x$ se ocorrer uma definição de $x$ no nó $n_{i}$ e existir um caminho livre de definição de $n_{i}$ para algum nó ou para algum arco que possua, respectivamente, um $c$-uso ou um $p$-uso de $x$. Um caminho $\left(n_{1}, n_{2}, \ldots, n_{k}\right)$ é um $d u$-caminho c.r.a variável $x$ se $n_{1}$ tiver uma definição global de $x$ e: (1) ou $n_{k}$ tem um c-uso de $x$ e $\left(n_{1}, n_{2}, \ldots, n_{j}, n_{k}\right)$ é um é um caminho simples livre de definição c.r.a $x$; ou (2) $\left(n_{j}, n_{k}\right)$ tem um $p$-uso de $x$ e $\left(n_{1}, n_{2}, \ldots\right.$, $\left.n_{j}, n_{k}\right)$ é um caminho livre de definição c.r.a $x$ e $n_{1}, n_{2}, \ldots, n_{j}$ é um caminho livre de laço [MAL91].

Os conjuntos utilizados para descrever os conceitos básicos dos critérios de testes para programas de aplicação são:

(i) $s$-uso $(i)=\left\{\right.$ variáveis com $s$-uso no nó $\left.n_{i} \in N_{s}\right\}$;

(ii) $\left.\operatorname{def} T<n_{\alpha}, n_{\beta}\right\rangle=\{$ Variável $\boldsymbol{t} \mid \boldsymbol{t}$ possui uma definição persistente pela concatenação dos nós $n_{\alpha}$ e $n_{\beta}$, representado por $<n_{\alpha}, n_{\beta}>$ onde $n_{\alpha}$ e $n_{\beta} \in N_{s}$; ; o nó $n_{\alpha}$ refere-se ao comando INSERT, UPDATE ou DELETE; o nó $n_{\beta}$ refere-se à confirmação da transação, pela ocorrência explícita ou implícita do comando COMMIT; a definição persistente é caracterizada quando o caminho executado possuir os nós $n_{\alpha}$ e $n_{\beta}$; 
(iii) $d s u(v, i)=\left\{\right.$ nós $n_{j} \in N_{s} \mid \boldsymbol{v} \in s$-uso $\left(n_{j}\right)$, existe um caminho livre de definição c.r.a. $\boldsymbol{v}$ do nó $n_{i}$ para o nó $n_{j}$ e $\left.\boldsymbol{v} \in \operatorname{defg}\left(n_{i}\right)\right\} ; \operatorname{defg}\left(n_{i}\right)$ representa o conjunto de todas as variáveis com definição global no nó $n_{i}$ )

(iv) t-uso $(j, k)=\left\{\right.$ Variável $\boldsymbol{t} \mid \boldsymbol{t}$ possui um uso nos $\operatorname{arcos}\left(n_{j}, n_{k}\right)$ sempre que um nó $n_{j}$ $\in N_{s}$ tiver um dos comandos INSERT, UPDATE, DELETE ou SELECT (arco de saída). O exemplo da Figura 2 possui um $\boldsymbol{t}$-uso nos $\operatorname{arcos}(2,3)$ e $(2,4)$ com relação a $\boldsymbol{t}$.

Dizemos que o caminho $\left(n_{\alpha}, \ldots, n_{\beta} \ldots, n_{j}, n_{k}\right)$ é um caminho livre de definição persistente do par de nós $\left\langle n_{\alpha}, n_{\beta}\right\rangle$ até o nó $n_{k}$, se não existir outro par de nós $\left\langle n_{q}, n_{m}>\right.$ onde ocorre uma redefinição persistente de $t \mathrm{em}<n_{q}, n_{m}>$ do nó $n_{\beta}$ até o nó $n_{k}$ onde o par $<n_{\alpha}, n_{\beta}>$ antecede o par $<n_{q}, n_{m}>$. Na ausência de um comando COMMIT, após um comando executável da SQL até o último nó de um grafo, adotou-se que o COMMIT estará no último nó do grafo. Essa é uma estratégia de implementação.

As seguintes definições complementam os conceitos básicos para a definição dos critérios de teste de programas de ABDR:

(v) Associação definição-t-uso é uma tripla $\left.\left[<n_{\alpha}, n_{\beta}\right\rangle,\left(n_{j}, n_{k}\right), \boldsymbol{t}\right]$ onde $\boldsymbol{t} \in$ $\operatorname{def} T<n_{\alpha}, n_{\beta}>, n_{j} \in d s u(t, \beta)$, o $\operatorname{arco}\left(n_{j}, n_{k}\right)$ é o arco de saída do nó $n_{\mathrm{j}}$.

(vi) dtu-caminho é um caminho livre de definição persistente $\left(n_{\alpha}, \ldots, n_{\beta}, \ldots, n_{j}, n_{k}\right)$ c.r.a. $\boldsymbol{t}$ dos nós $<n_{\alpha}, n_{\beta}>$ até o nó $n_{k}$ ou até o arco $\left(n_{j}, n_{k}\right)$ onde ocorre um uso de $\boldsymbol{t}$ e o caminho $\left(n_{\alpha}, \ldots, n_{\beta} \ldots, n_{j}, n_{k}\right)$, onde $n_{\beta}<n_{j}$ e $n_{\beta}<n_{k}$ é um caminho livre de laço e no par de nós $\left.<n_{\infty}, n_{\beta}\right\rangle$ ocorre uma definição persistente de $\boldsymbol{t}$. Um dtu-caminho é um du-caminho com relação à variável tabela no enfoque de variável persistente.

\section{Teste de Integração}

Esta seção introduz conceitos básicos de teste de integração, a partir de relações de fluxo de dados em variáveis tabela.

\subsection{Teste de integração baseado na dependência de dados}

A principal característica de programas de aplicação, que os distingue dos programas convencionais, é a persistência dos dados da base de dados. As unidades de programas e os módulos de programa, se autorizados, podem ter acesso a esses dados a qualquer momento. A persistência é uma propriedade que não existe exclusivamente em Banco de Dados Relacional (BDR), podendo existir para outros programas com características semelhantes.

Em ABDR, a mesma tabela da base de dados pode estar disponível para diferentes módulos de programa e, conseqüentemente, para diferentes UPS de um mesmo módulo. Neste caso, duas unidades de programa podem possuir uma dependência de dados entre elas, mesmo não existindo chamadas conectando-as.

Duas unidades de programa $U P_{A}$ e $U P_{B}$, não necessariamente distintas, possuem uma dependência de dados de $U P_{A}$ para $U P_{B}$ com relação à variável tabela $\boldsymbol{t}$, caso a unidade $U P_{A}$ possua um ponto no programa que define a variável tabela $t$ (isto é, altera o conteúdo da tabela cujo estado passa de $e_{i}$ para $e_{i+1}$ ) e a unidade de programa $U P_{B}$ possua um ponto no programa que usa a variável tabela $\boldsymbol{t}$ (com o estado de $\boldsymbol{t}$ igual a $\left.e_{i+1}\right)$. Uma variável tabela $\boldsymbol{t}$ com uma definição persistente em uma unidade $U P_{A}$ (passando a variável $\boldsymbol{t}$ para um estado $e_{A}$ ) estará em um estado consistente (representado por $e_{A}$ ) se e somente se não existir redefinição persistente de $t$ em pelo menos um subprograma $U P_{A}$ no nó de saída. Existe uma dependência de dados c.r.a. variável 
tabela $\boldsymbol{t}$ se $\boldsymbol{t}$ tiver uma definição persistente em uma unidade $U P_{A}$ e existir um caminho livre de definição c.r.a. $\boldsymbol{t}$ incluindo o sub-caminho de $U P_{A}$ a partir da definição persistente até o nó de saída e um sub-caminho do nó de entrada de uma unidade $U P_{B}$ até o arco $\left(n_{j}, n_{k}\right)$ que contém um $\boldsymbol{t}$-uso.

São considerados dois tipos de dependência de dados c.r.a $t$ :

i) Dependência interna ou intra-modular: ocorre quando existir uma dependência de dados entre unidades de programa de um mesmo Módulo, com relação a uma ou mais tabelas, mesmo quando não existir um ponto de chamada entre elas (Figura 3).

ii) Dependência externa ou inter-modular: ocorre quando existir uma dependência de dados entre duas ou mais UPS de Módulos diferentes c.r.a $\boldsymbol{t}$. Isto é, $\boldsymbol{t}$ deve estar disponível para os dois Módulos (Figura 4).

O Grafo de Dependência de dados de um programa de ABDR é denotado por

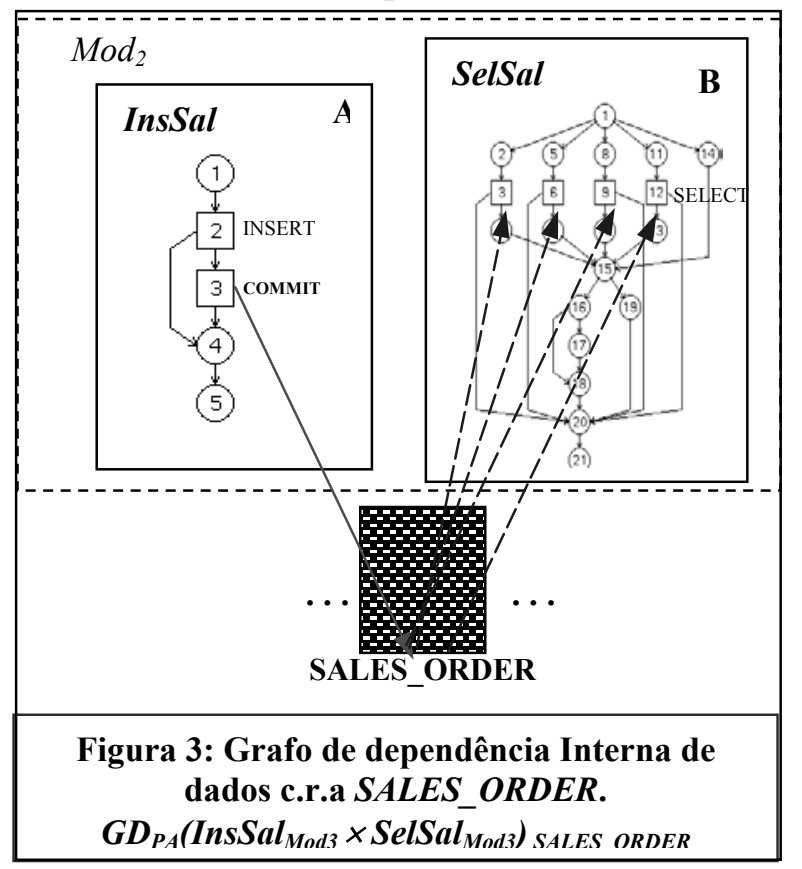

$\boldsymbol{G D}_{\boldsymbol{P A}}\left(\boldsymbol{A}_{\text {Modi }} \times \boldsymbol{B}_{\text {Modj }}\right)_{\boldsymbol{T}}=\left(\left[G\left(A_{i}\right) \times\right.\right.$ $\left.\left.G\left(B_{j}\right)\right], E_{\text {int }}, T\right)$, onde $G\left(\boldsymbol{A}_{i}\right) \times G\left(\boldsymbol{B}_{j}\right)$ representa a união dos conjuntos de arcos e nós do grafo de programa da unidade $\mathbf{A}$ do módulo de programa $\boldsymbol{i}$ e do grafo de programa da unidade $\mathbf{B}$ do módulo de programa $\boldsymbol{j} ; E$ é o conjunto de $\operatorname{arcos}$ (setas pontilhadas) que mostram o fluxo de dados das unidades de programa para a tabela e vice-versa, e $T$ é o conjunto de tabelas envolvidas na dependência de dados. $\boldsymbol{G D}_{P A}\left(\boldsymbol{A}_{M o d i} \times \boldsymbol{B}_{M o d j}\right)_{t}$ é um multi-grafo direcionado que representa o fluxo de dados baseado na dependência de dados extraída da associação def-uso c.r.a. t. Um exemplo desse grafo é apresentado na Figura 4.

Nas Figuras 3 e 4, uma seta (continua) direcionada da UP para a tabela

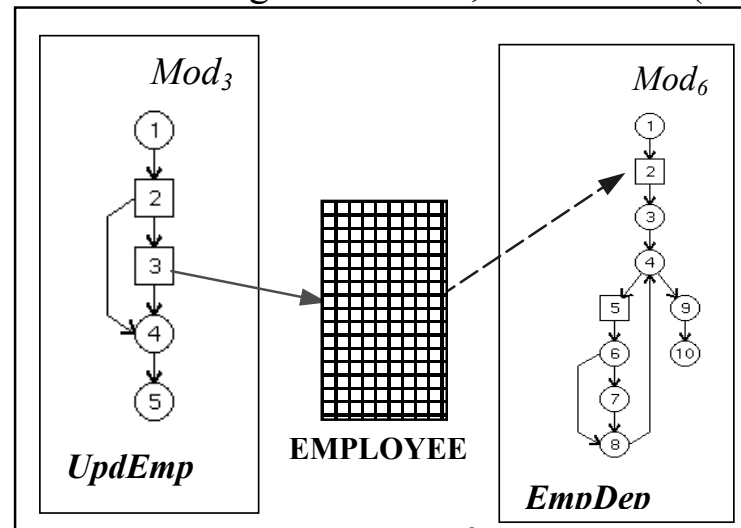

Figura 4: Grafo de dependência Externa de dados c.r.a EMPLOYEE entre as Ups UpdEmp $\left(\operatorname{Mod}_{3}\right)$ e EmpDep $\left(\operatorname{Mod}_{6}\right)$. $\operatorname{GD}_{\mathrm{PA}}\left(\text { UpdEmp }_{\mathrm{Mod} 3} \times \mathrm{EmpDep}_{\text {Mod6 }}\right)_{\text {EMPLOYEE }}$ caracteriza a ocorrência de uma definição persistente da variável tabela $\boldsymbol{t}$ pela UP; uma seta (pontilhada) direcionada da tabela $t$ para a $U P$ caracteriza a ocorrência de um uso da tabela $t$ pela $U P$. O exemplo mostrado na Figura 3 é um grafo de dependência Interna, contudo se as UPS $A$ e $B$ pertencerem a Módulos de Programas distintos tratar-se-á de grafo de dependência Externa (Figura 4). No exemplo da Figura 3, a UP InsSal define a variável tabela SALES_ORDER (nó 3) e a UP SelSal usa a variável tabela SALES_ORDER em vários pontos do programa (nós 3, 6 e 9). Isso é feito para todas as UPs que possuem uma definição persistente, combinadas com todas as UPS que usam a mesma variável tabela. Já no exemplo da Figura 4 a UP UpdEmp do Módulo de Programa $\operatorname{Mod}_{3}$ define a variável 
tabela EMPLOYEE no nó 3 e a UP EmpDep do Módulo de Programa $\operatorname{Mod}_{6}$ usa essa mesma variável no nó 2 .

\section{Tipos de Fluxos de Dados}

A análise de fluxo de dados para programas de ABDR, que é composta por dois tipos de Fluxos de Dados - intra-modular e inter-modular - explora as associações persistentes geradas pelas variáveis tabela. Esta seção apresenta as definições básicas e a terminologia para a definição de critérios de teste estrutural de integração que enfocam as variáveis tabela utilizadas em programas de ABDR.

\subsection{Fluxo Intra-modular}

O fluxo de dados intra-modular é utilizado para o teste de integração de cada Módulo de Programa. Inicialmente é feito o teste de cada UP que compõe o Módulo de Programa (teste intra-unidade ou teste de unidade) [SPO00a]. Depois que todas as

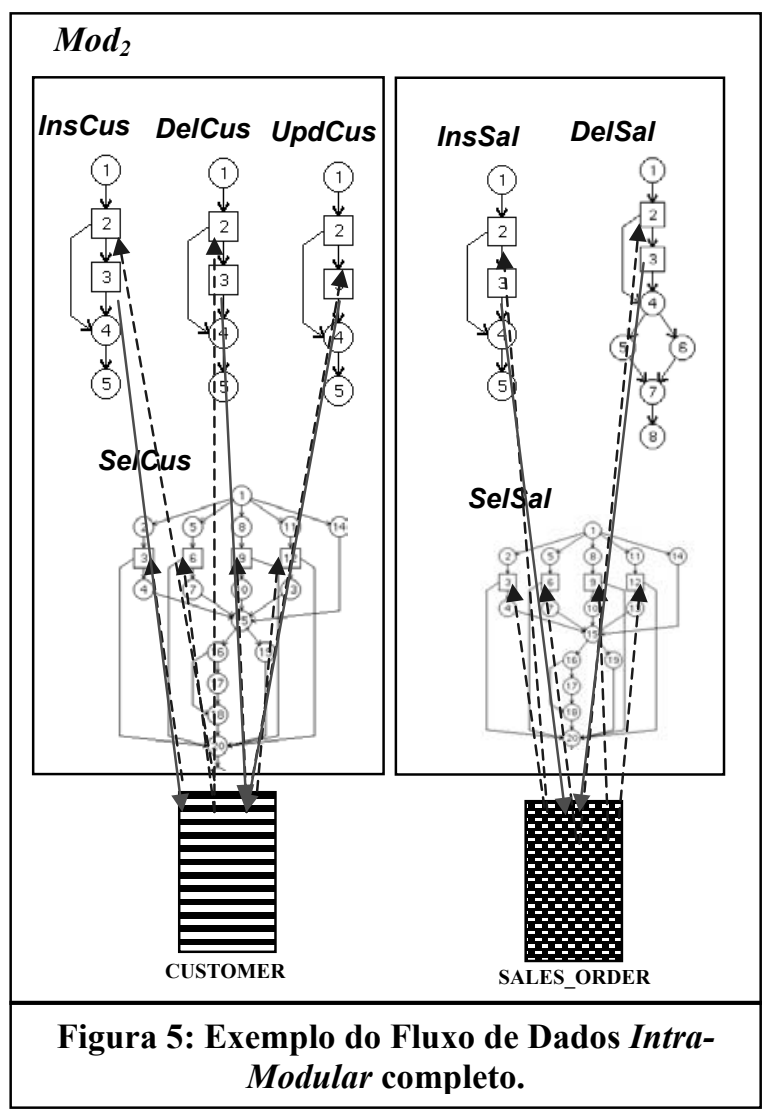

Unidades foram devidamente testadas, realiza-se o teste de integração para as UPs do Módulo de Programa (teste inter-unidades ou teste de integração intra-modular).

A partir do grafo de programa $G(U P)$ de cada $U P$ do Módulo é construído o grafo de dependência de dados que representa os fluxos de dados entre as UPs que compõem o Módulo. $\mathrm{O}$ grafo de dependência baseia-se na hierarquia de chamada entre as UPs ou na dependência de dados entre as UPs determinada pelas variáveis tabela presentes no Módulo.

A Figura 5 apresenta um exemplo completo de dependência de dados entre as unidades de um mesmo módulo $\left(\operatorname{Mod}_{2}\right)$. O Módulo $\operatorname{Mod}_{2}$ possui comandos de SQL com definições e usos persistentes de duas das oito variáveis tabela mostradas na Figura 6. Toda seta direcionada da variável tabela $\boldsymbol{t}$ para o nó de um grafo representa um uso de $\boldsymbol{t}$ naquele nó e toda seta apontada do nó para a variável tabela $\boldsymbol{t}$ representa uma definição persistente de $\boldsymbol{t}$ naquele nó. Toda unidade que tem um uso de uma variável $\boldsymbol{t}$ possui uma dependência de dados com as unidades que têm uma definição persistente da mesma variável $\boldsymbol{t}$.

\subsection{Fluxo Inter-modular}

O fluxo de dados inter-modular é determinado a partir das dependências de dados ocasionadas pelas tabelas da base de dados comuns aos Módulos da ABDR. A unidade $U P_{i}$ no Módulo $\operatorname{Mod}_{k}$ pode estar definindo uma variável tabela $t$ enquanto a unidade $U P_{j}$ contida no Módulo $\operatorname{Mod}_{W}, K \neq W$, pode estar usando a mesma variável tabela $\boldsymbol{t}$, 
determinando, assim, um fluxo de dados entre os módulos $\operatorname{Mod}_{k}$ e $\operatorname{Mod}_{w}$. Assim, é possível definir associações definição-uso c.r.a $\boldsymbol{t}$ (denominada de associação definiçãot-uso-inter) entre os módulos de programas criando um grafo de dependência externa de dados entre a $U P_{i}$ do Módulo $\operatorname{Mod}_{k}$, que possui uma definição persistente da variável $\boldsymbol{t}$, e a $U P_{j}$ do Módulo $\operatorname{Mod}_{w}$, que possui um uso da variável $\boldsymbol{t}$. A representação gráfica do modelo de fluxo de dados inter-modular é composta por nós retangulares contínuos que representam os Módulos de Programa e nós retangulares pontilhados, representando as variáveis tabela que compõem a base de dados da aplicação. As setas indicam os fluxos de dado entre os nós. Tendo em vista que um Módulo de Programa é visto como um conjunto de Unidades de Programa $\operatorname{Mod}_{a}=\left\{U P_{1}, . ., U P_{m}\right\}$, temos:

a) Seta do Módulo de Programa $\left(\operatorname{Mod}_{i}\right)$ para tabela $t$ : indica que existe pelo menos uma $U P_{\alpha} \subset \operatorname{Mod}_{i}$ tal que $U P_{\alpha}$ tem uma definição persistente da tabela $\boldsymbol{t}$;

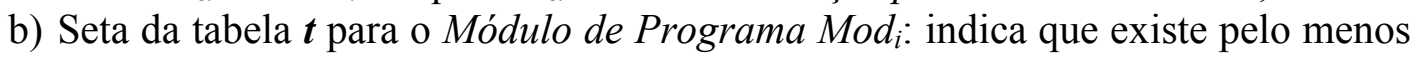
uma $U P_{\alpha} \subset \operatorname{Mod}_{i}$ tal que $U P_{\alpha}$ tem um uso persistente da tabela $\boldsymbol{t}$.

No fluxo inter-modular, a execução de cada módulo de programa é feita isoladamente, não havendo, portanto, rotinas de controle entre eles. De acordo com o exemplo apresentado na Figura 6, o módulo de programa $\operatorname{Mod}_{2}$ define e usa as tabelas CUSTOMER e SALES_ORDER. É possível determinar as associações definição-uso inter-modular para cada Módulo de Programa $(M o d)$; essas informações são extraídas das UPs pertencentes a cada Módulo de Programa que possuírem um par $\left\langle n_{\infty}, n_{\beta}\right\rangle$, da definição persistente de $\boldsymbol{t}$, com as UPs que possuírem um comando da SQL que usa a tabela $\boldsymbol{t}$.

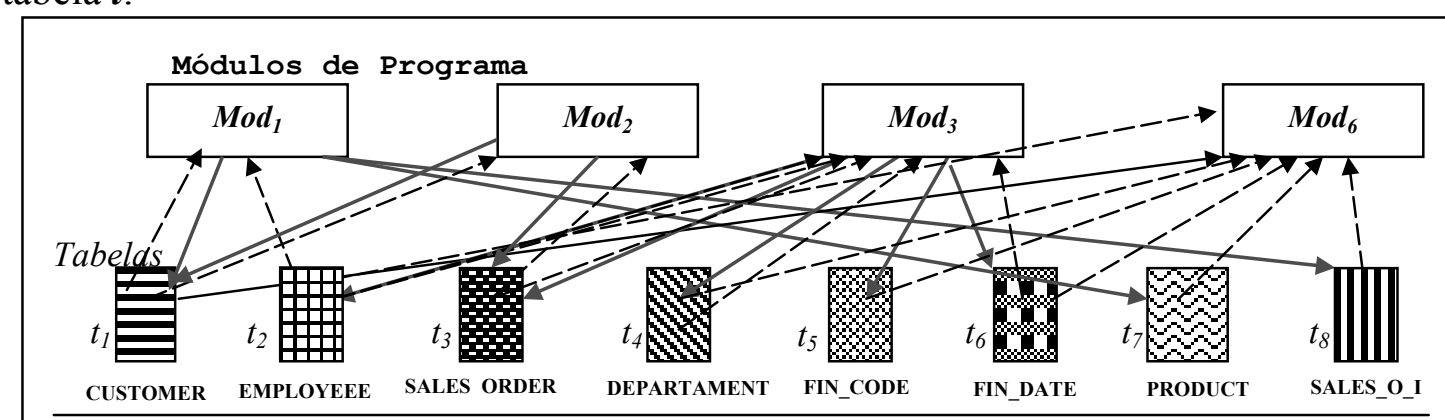

Figura 6: Fluxo de dados inter-modular de uma ABDR composta por 4 Módulos de Programa e 8 tabelas aue constituem a base de dados

Tabela 1: Mapa de definição e uso das variáveis $t_{i}$ mostradas nos Módulos de Programas da Figura 6

\begin{tabular}{|c|c|c|c|c|c|c|c|c|c|c|c|c|c|c|c|c|}
\hline \multirow{2}{*}{ Módulos de Programa } & \multicolumn{8}{|c|}{ Tabelas definidas } & \multicolumn{8}{|c|}{ Tabelas usadas } \\
\hline & $t_{1}$ & $t_{2}$ & $t_{3}$ & $t_{4}$ & $t_{5}$ & $t_{6}$ & $t_{7}$ & $t_{8}$ & $t_{1}$ & $t_{2}$ & $t_{3}$ & $t_{4}$ & $t_{5}$ & $t_{6}$ & $t_{7}$ & $t_{8}$ \\
\hline $\operatorname{Mod}_{1}$ & $\mathrm{X}$ & $\mathrm{X}$ & & & & & $\mathrm{X}$ & $\bar{X}$ & $\mathrm{X}$ & & & & & & & \\
\hline $\mathrm{Mod}_{2}$ & $\mathrm{X}$ & & $\mathrm{X}$ & & & & & & $\mathrm{X}$ & & $\mathrm{X}$ & & & & & \\
\hline $\mathrm{Mod}_{3}$ & & $\mathrm{X}$ & $\mathrm{X}$ & $\mathrm{X}$ & $\mathrm{X}$ & $\mathrm{X}$ & & & & $\mathrm{X}$ & $\mathrm{X}$ & $\mathrm{X}$ & $\mathrm{X}$ & $\mathrm{X}$ & & \\
\hline $\operatorname{Mod}_{6}$ & & & & & & & & & $\mathrm{X}$ & $\mathrm{X}$ & $\bar{X}$ & $\mathrm{X}$ & $\mathrm{X}$ & $\mathrm{X}$ & $\mathrm{X}$ & $\bar{X}$ \\
\hline
\end{tabular}

Para melhor entendimento da Figura 6, é apresentado na Tabela 1 um mapa que relaciona para cada Módulo de Programa, as ações de ocorrência (definição e uso) para cada variável tabela (numeradas da esquerda para a direita).

\subsection{Os critérios de integração intra-modulares}

Os critérios de teste intra-modulares são aplicados no teste de cada UP do Módulo (teste de unidade) e no teste de integração das UPs do Módulo. Para o teste de unidade 
podemos utilizar qualquer critério de teste estrutural. Os critérios para o teste de integração são divididos em dois subgrupos: critérios de testes baseados no grafo de chamadas e critérios de teste baseados na dependência de dados das tabelas.

Os critérios de teste propostos para associar as variáveis persistentes devem ser satisfeitos com a mesma tupla, forçando o testador a gerar casos de testes específicos para exercitá-la. Deste modo, o teste deve ser executado de maneira controlada, não sendo suficiente usar qualquer tupla de $t$. Por outro lado, a exigência do uso da mesma tupla para satisfazer uma associação definição-t-uso, pode aumentar o número de elementos requeridos não factíveis (isto é, não executáveis com a mesma tupla).

\section{$\checkmark$ Critérios de teste de integração baseados na dependência de dados}

Os critérios de integração intra-modular visam a exercitar as associações definição-t-uso determinadas pelos comandos de manipulação da base de dados. Os critérios de integração baseados na dependência de dados determinada pelas tabelas da base de dados visam a exercitar as associações referentes às variáveis de programa $(\boldsymbol{P})$, variáveis host $(\boldsymbol{H})$ ou variáveis tabela $(\boldsymbol{T})$, de acordo com o enfoque estabelecido pelos critérios.

A variável tabela definida por uma unidade de programa mantém-se viva ao longo do tempo, determinando a existência de uma associação def-t-uso entre duas UPS executadas em momentos distintos, sem a necessidade de um comando de chamada de uma para outra. Dizemos que uma associação def-t-uso existe, se uma variável $t$ definida em $U P_{A}$ tem um $t$-uso em $U P_{B}$.

As associações definição-t-uso de integração são exercitadas com seqüências de execuções para pares de unidades ( $U P^{d}$, unidade que define $\boldsymbol{t}$; $U P^{u}$, unidade que usa $\boldsymbol{t}$ ). Neste caso, é importante definir uma estratégia que exercite essas associações, utilizando os mesmos valores, tanto para a execução de $U P^{d}$ como para a de $U P^{u}$.

\section{$\checkmark$ Critérios baseados no fluxo de dados intra-modular}

Estes critérios baseiam-se nas dependências de dados existentes entre os procedimentos de um mesmo Módulo de Programa com relação a uma variável tabela da base de dados e requerem a mesma tupla para satisfazer a associação definição-t-uso. Cada unidade $U P_{A}$ que contém uma definição persistente da variável $\boldsymbol{t}$ é associada a outra unidade $U P_{B}$ que contém um uso de $\boldsymbol{t}\left(\boldsymbol{t}\right.$-uso); os pares $\left(U P_{A}, U P_{B}\right)$ são requeridos pelo critério com um ciclo de execução (denomina-se de ciclol). A noção de ciclo 1 denota que somente as UPs que definem a associação serão executadas para cobrir a associação; em alguns casos, como dependência por chave estrangeira, pode ser que outras UPs necessitem ser executadas, requerendo mais ciclos de execução para cobrir a associação.

Considere que $G\left(U P_{A}\right)$ e $G\left(U P_{B}\right)$ sejam os grafos das unidades A e B, respectivamente, envolvidas na integração e $\Pi$ seja o conjunto dos caminhos completos em $G\left(U P_{A}\right)$ e $G\left(U P_{B}\right)$ e $\Gamma$ o conjunto de tuplas. $\left(\pi_{a}, \pi_{b}\right) \in \Pi$ implica que existe a concatenação $\pi_{a}, \pi_{b}$, onde $\pi_{a} \in G\left(U P_{A}\right)$ e $\pi_{b} \in G\left(U P_{B}\right)$.

Todas os t-usos-ciclo1-intra: $\Pi$ e $\Gamma$ satisfazem o critério todos os t-usos-ciclo1intra para o par de unidades $U P_{A}$ e $U P_{B}$ pertencentes ao mesmo módulo se, para todos os pares de caminhos $\left(\pi_{a}, \pi_{b}\right) \in \Pi: \pi_{a}$ incluir o par de nós $<n_{\alpha}, n_{\beta}>\in G\left(U P_{A}\right)$ tal que $\operatorname{def} T<n_{\alpha}, n_{\beta}>\neq \phi$ c.r.a. $\boldsymbol{t}$; existir um caminho livre de definição c.r.a. $\boldsymbol{t}$ de $<n_{\alpha}, n_{\beta}>$ até $n_{\text {out }}$ de $G\left(U P_{A}\right) ; \pi_{b}$ incluir o $\operatorname{arco}\left(n_{j}, n_{k}\right) \in G\left(U P_{B}\right)$ onde $j \in f d s u\left(t, n_{\beta}\right)$; existir um $\boldsymbol{t}$-uso 


\section{Simpósio Brasileiro de Qualidade de Software}

em $\left(n_{j}, n_{k}\right)$; e existir um caminho livre de definição persistente c.r.a. $\boldsymbol{t}$ de $n_{\text {in }}$ até o arco $\left(n_{j}, n_{k}\right)$; a associação é satisfeita se for exercitada através da mesma tupla $\tau \in \Gamma$.

Uma associação-t-uso-ciclol-intra para o módulo $z$ é representada pela quíntupla:

$$
\left[{ }^{z} U P_{A},<n_{\alpha}, n_{\beta}>,{ }^{\mathrm{z}} U P_{B},\left(n_{j}, n_{k}\right), \boldsymbol{t}\right]
$$

Todos os dtu-caminhos-intra: $\Pi$ e $\Gamma$ satisfazem o critério todos os dtucaminhos-intra para o par de unidades $U P_{A}$ e $U P_{B}$ pertencentes ao mesmo módulo se, para todos os pares de caminhos $\left(\pi_{a}, \pi_{b}\right) \in \Pi$ : $\pi_{a}$ incluir um dtu-caminho do nó $n_{\alpha}$ até o nó $n_{\text {out }}$ de $G\left(U P_{A}\right)$ passando pelo par de nós $\left.<n_{\alpha}, n_{\beta}\right\rangle \subseteq G\left(U P_{A}\right)$ onde $\operatorname{def} T<n_{\omega}, n_{\beta}>\neq \phi$ c.r.a. $\boldsymbol{t}$, através da tupla $\tau$, $\pi_{b}$ incluir um dtu-caminho c.r.a $\boldsymbol{t}$ do nó $n_{\text {in }}$ de $G\left(U P_{B}\right)$ até $o$ $\operatorname{arco}\left(n_{j}, n_{k}\right) \in G\left(U P_{B}\right)$ sendo $n_{j} \in f d s u\left(t, n_{\beta}\right)$; existir um $\boldsymbol{t}$-uso em $\left(n_{j}, n_{k}\right)$, através da mesma tupla $\tau$; e existir um caminho livre de definição persistente que vai do par $<n_{\phi}$ $n_{\beta}>$ em $U P_{A}$ até o $\operatorname{arco}\left(n_{j}, n_{k}\right)$ em $U P_{B}$ c.r.a $t$ na composição dos caminhos $\pi_{a} \cdot \pi_{b}$.

O elemento requerido pelo critério todos os dtu-caminhos-intra é representado pela n-upla abaixo, sendo $z$ o número do módulo que contém as unidades $A$ e $B$ :

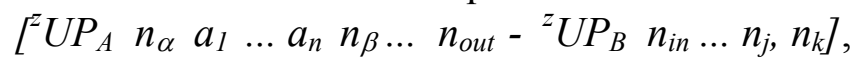

onde ${ }^{z} U P_{A}$ é a unidade onde ocorre a definição de $t$ e $n_{\alpha} a_{l} \ldots a_{n} n_{\beta} \ldots n_{\text {out }}$ é o dtucaminho de $<n_{\alpha}, n_{\beta}>$ até o nó de saída, podendo ou não passar por um sub-caminho $\left(a_{1}\right.$ $\left.\ldots a_{n}\right)$ livre de laço; ${ }^{z} U P_{B}$ é a unidade onde ocorre o uso de $\boldsymbol{t}$ e $n_{i n} \ldots n_{j} n_{k}$ é o dtucaminho que vai do nó inicial até o $\operatorname{arco}\left(n_{j}, n_{k}\right)$ onde existe um $t$-uso c.r.a.t.

Existem dependências que não podem ser exercitadas com a execução de apenas duas unidades (uma de definição e outra de uso), mas necessitam da execução de outra unidade para satisfazer a associação. Isso ocorre com a existência da integridade referencial entre duas tabelas, ou seja, para definir a variável $\boldsymbol{t}$ é necessário estar definida a variável $\boldsymbol{t}$ ' e, para isso, pode ser necessário executar a unidade que define a variável $\boldsymbol{t}$ ' para depois executar a unidade que define $\boldsymbol{t}$ e finalmente executarmos a $U P$ que usa $t$.

Todos os t-usos-ciclo2-intra: $\Pi$ e $\Gamma$ satisfazem o critério (todos os $t$-usos-ciclo2intra) para as unidades $U P_{A}, U P_{B}$ e $U P_{C}$ pertencentes ao mesmo módulo se, para todos os caminhos $\left(\pi_{a}, \pi_{b}, \pi_{c}\right) \in \Pi$, onde $\pi_{a}$ é o caminho que contém um par de nós $<n_{\alpha^{\prime}}, n_{\beta^{\prime}}>$ que define a variável $\boldsymbol{t}$ ' pela tupla $\tau$ '; o caminho $\pi_{b}$ contém um par de nós $\left.<n_{\alpha}, n_{\beta}\right\rangle$ que define $\boldsymbol{t}$ pela tupla $\tau$ podendo ou não ter uma dependência de $\boldsymbol{t}^{\prime}$, $e$ o caminho $\pi_{c}$ possuir um nó que contém um uso de $\boldsymbol{t}$ e ou $\boldsymbol{t}$ ' pela mesma tupla $\tau$ e/ou $\tau^{\prime}$, respectivamente, e o caminho $\pi_{a}$ passar pelo par de nós $<n_{\alpha^{\prime}}, n_{\beta^{\prime}}>\in \mathrm{G}\left(U P_{A}\right) \operatorname{com} \operatorname{def} T<n_{\alpha^{\prime}}, n_{\beta^{\prime}}>\neq \phi$ c.r.a. $t^{\prime}$ : existir um caminho livre de definição c.r.a. $t$ ' de $n_{\beta}$ até $n_{\text {out }}$ de $G\left(U P_{A}\right)$; o caminho $\pi_{a}$ passar pelo par de nós $<n_{\alpha}, n_{\beta}>\in \mathrm{G}\left(U P_{B}\right)$ onde $\operatorname{def} T<n_{\alpha}, n_{\beta}>\neq \phi$ c.r.a. $t$; existir um caminho livre de definição c.r.a. $t$ de $n_{\beta}$ até $n_{\text {out }}$ de $G\left(U P_{B}\right)$; o caminho $\pi_{b}$ passar pelo $\operatorname{arco}\left(n_{j}, n_{k}\right) \in G\left(U P_{C}\right) /$ existe um $\boldsymbol{t}$-uso em $\left(n_{j}, n_{k}\right)$ e $n_{j} \in f d s u\left(\boldsymbol{t}, n_{\beta}\right)$ (sendo $\boldsymbol{t}$ e/ou $\boldsymbol{t}$ ') e existir um caminho livre de definição c.r.a. $\boldsymbol{t}$ e/ou $\boldsymbol{t}$ ' de $n_{i n}$ até o $\operatorname{arco}\left(n_{j}, n_{k}\right)$. A associação $\left.\left.\left[<n_{\alpha^{\prime}}, n_{\beta^{\prime}}\right\rangle, U P_{A},<n_{\omega} n_{\beta}\right\rangle, U P_{\boldsymbol{B}},\left(n_{j}, n_{k}\right), U P_{C},\left\{\boldsymbol{t}^{\prime}, \boldsymbol{t}\right\}\right]$ é satisfeita se e somente se a mesma tupla $\tau\left(\tau^{\prime}\right)$ usada para satisfazer a definição de $\boldsymbol{t}$ ( $\boldsymbol{t}^{\prime}$ ) é também usada para satisfazer o uso.

Neste caso, os elementos requeridos são representados por uma sétupla do tipo:

$$
\left[{ }^{z} U P_{A},<n_{\alpha}, n_{\beta}>,{ }^{z} U P_{B},<n_{\alpha^{\prime}}, n_{\beta^{\prime}}>,{ }^{\mathrm{z}} U P_{C},\left(n_{j}, n_{k}\right),\left\{\boldsymbol{t}, \boldsymbol{t}^{\boldsymbol{\gamma}}\right\}\right]
$$


Dois enfoques podem ser considerados: utilizar as mesmas tuplas para satisfazer os pares definição-uso na integração ou utilizar tuplas diferentes para satisfazer os elementos requeridos não cobertos com a mesma tupla. $O$ primeiro enfoque é mais conservador e mostrou, na execução de um exemplo de aplicação, ser melhor para a detecção de defeitos do que o segundo; contudo, este enfoque possui maior custo associado, pois a quantidade de elementos requeridos tende a ser maior.

\subsection{Critérios de integração inter-modular}

Os critérios de integração inter-modular são semelhantes aos critérios de integração intra-modular com a diferença de que as unidades associadas devem pertencer a Módulos distintos. Esses critérios visam a exercitar associações de variáveis tabela que são definidas em unidades de um Módulo $\alpha$ e são usadas em unidades de outro Módulo $\beta$.

Considere duas unidades $U P_{A}$, pertencente a um módulo $\operatorname{Mod}_{x}$, e $U P_{B}$, pertencente a outro módulo $\operatorname{Mod}_{y}$. Considere também os mesmos conjuntos $\Pi$ e $\Gamma$ definidos anteriormente. Temos:

Todos os t-usos-ciclo1-inter: Este critério é similar ao critério todos os t-usosciclo1-intra, com a única diferença que as unidades $U P_{A}$ e $U P_{B}$ pertencem a Módulos distintos. Neste caso o elemento requerido é representado pela quíntupla:

$$
\left[{ }^{z} U P_{A},<n_{\omega}, n_{\beta}>,{ }^{w} U P_{B},\left(n_{j}, n_{k}\right), \boldsymbol{t}\right]
$$

onde $z$ representa o módulo $\operatorname{Mod}_{z}$ e $w$ representa $\operatorname{Mod}_{w}$.

Todos os dtu-caminhos-inter: Este critério é similar ao critério todos os dtucaminhos-intra, com a única diferença que a unidade onde existe uma definição persistente $\left(U P_{A}\right)$ pertence a um Módulo e a unidade onde existe um $\boldsymbol{t}$-uso pertence a outro Módulo. Neste caso o elemento requerido é representado pela quíntupla:

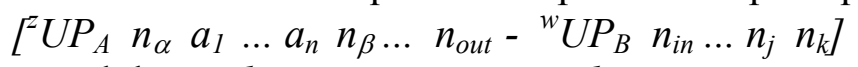

onde $z$ representa o módulo $\operatorname{Mod}_{z}$ e $w$ representa $\operatorname{Mod}_{w}$.

Todos os t-usos-ciclo2-inter: Este critério é similar ao critério todos os t-usosciclo2-intra, com a diferença que as unidades associadas devem pertencer a Módulos distintos. Neste caso, os elementos requeridos são representados por uma sétupla do tipo:

$$
\left[{ }^{z} U P_{A},<n_{\alpha}, n_{\beta}>,{ }^{y} U P_{B},<n_{\alpha^{\prime}}, n_{\beta^{\prime}}>,{ }^{\mathrm{w}} U P_{C},\left(n_{j}, n_{k}\right),\left\{\boldsymbol{t}, \boldsymbol{t}^{\prime}\right\}\right]
$$

\subsection{Aplicação dos Critérios Propostos}

Os critérios propostos foram utilizados no teste de integração em aplicações reais, para revelar defeitos existentes e semeados em laboratório. Para tal, a Ferramenta POKETOOL [CHA91], originalmente voltada à automação de teste de fluxo de dados de programas convencionais, foi adaptada para apoiar a aplicação dos critérios de integração para ABDRs.

Para exemplificar, considere o teste de integração intra-modular baseado na dependência de dados, para a variável tabela EMPLOYEE no Módulo de Programa denominado de Mod3. Os elementos requeridos dos critérios todos os t-usos-ciclo1intra e todos os dtu-caminhos-intra são mostrados na Figura 7.

Para ilustrar, $<\mathbf{3 0 0 1},<\mathbf{2 , 3}>, \mathbf{3 0 1 6},(\mathbf{2 , 4}),\{$ EMPLOYEE $\}>$ é a associação entre as unidades 3001 e 3016; no par $<\mathbf{2 , 3}>$ ocorre a definição persistente da variável EMPLOYEE, na unidade 3001; e no arco $(2,4)$ ocorre o uso da variável EMPLOYEE 
na unidade 3016. O mesmo ocorre com os elementos requeridos para o critério todos os dtu-caminhos-intra representados pelas seqüências $\begin{array}{lllllllllll}3011 & 2 & 3 & 4 & 5 & 3016 & 1 & 11 & 12\end{array}$ 18; a primeira é o dtu-caminho $(2,3,4,5)$ na unidade 3011 referente à definiçãopersistente de EMPLOYEE; a segunda é o dtu-caminho $(\mathbf{1}, \mathbf{1 1}, \mathbf{1 2}, \mathbf{1 8})$ na unidade 3016 referente ao uso da variável persistente.

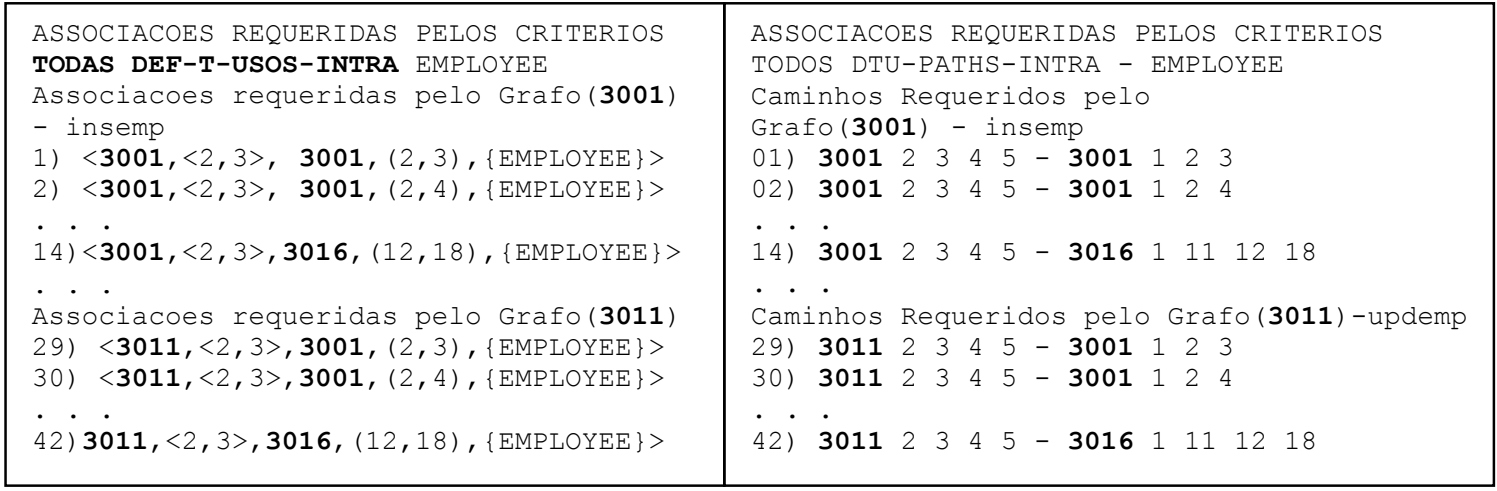

Figura 7: Elementos requeridos pelos critérios todos os t-usos-ciclo1-intra e todos os dtucaminhos-intra.

Na Figura 8 enumeram-se os caminhos percorridos pela aplicação dos casos de teste e o valor da chave primária ou conjunto de chaves utilizadas em cada execução. Por exemplo, as execuções dos casos de testes \#2 e \#3 exercitaram o elemento requerido 15 , mencionado na Figura 7, do critério todos-t-usos-ciclol-intra, onde em ambos foi manipulada a mesma tupla, identificada por 1900 .

A Tabela 2 apresenta o total de elementos requeridos (elem.req.) pelo critério Todos os t-usos-ciclol-intra, para as variáveis tabela associadas ao Módulo Mod3.

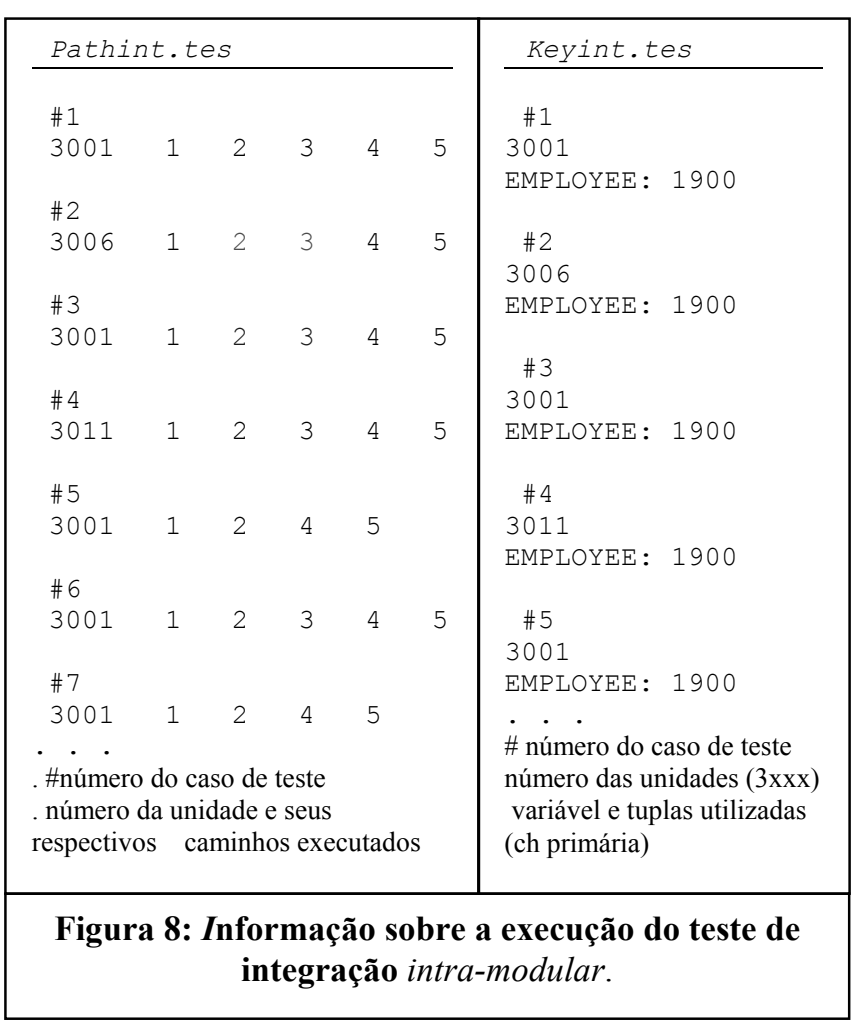
Apresenta também o total de elementos requeridos não exercitáveis (ne) e o número de casos de teste para exercitar os elementos requeridos exercitáveis. A Tabela 3 mostra o resultado para tabelas associadas nos testes de integração (intra-modular e intermodular) dos Módulos para os critérios de ciclo1 e ciclo2.

Não existe elemento requerido para o teste de integração intra-modular do Módulo $\operatorname{Mod}_{6}$, tendo em vista que este programa emite apenas relatórios e usa diversas tabelas em seu código de SQL. Na integração inter-modular, existem 48 elementos re-queridos em cada ciclo para o critério todos os t-usos (sendo $42 \mathrm{com} \mathrm{Mod}_{3}$ e 6 com o $\operatorname{Mod}_{2}$ ). Os defeitos ocasionados pelas queries durante as consultas só foram observados com a integração entre os módulos que associaram com o $\operatorname{Mod}_{6}$. 
O número total (234) de casos de teste para o teste de integração (critério todost-usos-ciclo1-intra) do Módulo $\operatorname{Mod}_{3}$ (Tabela 2), necessário para exercitar os elementos requeridos, deve-se à exigência de uso da mesma tupla no critério de teste de integração.

Unidades de programas que somente emitem relatórios (possuem apenas comandos SELECT) não tiveram elementos requeridos durante o teste de unidade no escopo de variáveis persistentes. Contudo, na combinação de unidades, foram identificados elementos requeridos pelos critérios de integração.

Foi observado que os critérios de integração intra-modular e de integração intermodular não podem ser comparados quanto a sua força relativa, ou seja, quanto à dificuldade em satisfazê-los. Em adição, tais critérios enfocam aspectos distintos de programas de uma ABDR, tornando-os, então, incomparáveis teoricamente. A aplicação dos critérios Potenciais Usos não destaca os defeitos referentes ao uso dos comandos da SQL, sendo estes observados somente com os critérios baseados na dependência de dados intra-modular e inter-modular.

Tabela 2: Testes de integração critério todos os t-usos-ciclo1-intra para o $\operatorname{Mod}_{3}$

\begin{tabular}{|c|c|c|}
\hline Tabelas & Total de elem.req. (ne) & $\boldsymbol{N}^{\text {os }}$ de Casos de testes \\
\hline Departamento & $36(9)$ & 60 \\
\hline Employee & $42(10)$ & 65 \\
\hline Fin & $48(24)$ & 60 \\
\hline Sales_Order & $36(10)$ & 49 \\
\hline
\end{tabular}

Tabela 3: Elementos requeridos pelo critério todos os t-usos-inter $\left(\operatorname{Mod}_{2}, \operatorname{Mod}_{3}\right.$ e $\left.\operatorname{Mod}_{6}\right)$

\begin{tabular}{|l|l|c|c|c|c|}
\hline Mod def & Mod $_{\text {uso }}$ & \multicolumn{2}{|c|}{$\begin{array}{c}\text { Elementos } \\
\text { Requeridos } \\
\text { Todos os } \text {-usos }\end{array}$} & \multicolumn{2}{c|}{ Casos de testes } \\
\cline { 3 - 6 } & & Ciclo1 & ciclo2 & ciclo1 & Ciclo2 \\
\hline $\operatorname{Mod}_{2}$ & $\operatorname{Mod}_{3}$ & $\mathbf{9}$ & - & $\mathbf{1 8}$ & - \\
\hline $\operatorname{Mod}_{2}$ & $\operatorname{Mod}_{6}$ & $\mathbf{6}$ & $\mathbf{6}$ & $\mathbf{1 2}$ & $\mathbf{1 8}$ \\
\hline $\operatorname{Mod}_{3}$ & $\operatorname{Mod}_{2}$ & $\mathbf{9}$ & - & $\mathbf{1 8}$ & - \\
\hline $\operatorname{Mod}_{3}$ & $\operatorname{Mod}_{6}$ & $\mathbf{4 2}$ & $\mathbf{4 2}$ & $\mathbf{4 0}$ & $\mathbf{6 0}$ \\
\hline
\end{tabular}

\section{Conclusões}

Foram exploradas as relações de fluxo de dados persistentes, em aplicações de banco de dados relacional (ABDR), para abstrair requisitos de teste estrutural de integração. $O$ objetivo foi suprir a carência de critérios de teste estrutural de integração para ABDR, envolvendo o uso da SQL (structured query language) para a manipulação de dados persistentes.

Foi proposta uma abordagem aos testes estruturais de integração intra-modular e inter-modular em programas com SQL embutida. Tais critérios são baseados na estrutura de chamada entre unidades e na dependência exclusiva de dados, ambos focados nas relações de fluxo de dados persistentes.

A criação de novas estratégias de teste estrutural, visando a exercitar aplicações de Banco de Dados, complementa as técnicas de teste estrutural já existentes na literatura e os novos critérios contribuem com novas abordagens de teste que podem ser aplicadas a variáveis persistentes de um modo geral. Os critérios propostos, ao exigirem o uso da mesma tupla para satisfazer as associações definição-t-uso, distinguem-se da maioria dos demais critérios de teste; isto obriga o testador a escolher os dados de teste com mais cuidado e resulta, ao mesmo tempo, em um teste mais efetivo no que se refere à integridade das tabelas. 
A partir de exemplos de aplicação foi possível observar que os critérios são complementares, pois possuem requisitos próprios por abordar estruturas e conceitos distintos, tais como diferentes tipos de fluxo de dados. Em adição, dependendo da natureza da integração entre unidades e módulos existentes na aplicação, o emprego de uma das abordagens de integração pode ser suficiente durante a atividade de teste; por exemplo, o $\operatorname{Mod}_{6}$ (ver Figura 6), o qual é um programa que usa somente as variáveis tabela, pode ter todos os seus comandos SQL exercitados com o teste de integração inter-modular mostrado na Figura 4.

A realização de estudos experimentais controlados, visando a avaliar o custo, a aplicabilidade e a propensão em revelar defeitos dos critérios propostos, será o próximo passo direcionado à melhoria da qualidade de ABDRs.

Agradecimento: Agradecemos ao $C N P q$ e Capes pelo apoio financeiro.

\section{Referências}

[CHA91] Chaim, M. L. "Poke-tool - Uma Ferramenta para Suporte ao Teste Estrutural de Programas Baseado em Análise de Fluxo de Dados", Dissertação de Mestrado, DCA/FEE/UNICAMP, Campinas - SP - Brasil, Abril 1991.

[CHA00] Chays, D., Dan, S., Frankl, P.G., Vokolos, F.I., Weyuker, E.J. “A Framework for Testing Database Applications". Proceedings of the ISSTA'00-, Agosto, 2000, pp. 147-157.

[CHA03] Chays, D., Deng, Y., "Demonstration of AGENDA Tool Set for Testing Relational Database Applications", Proc. of The Intl. Symposium on Software Engineering, Porland, Oregon, 2003.

[CLA89] Clarke, L. A., Podgurski, A., Richardson, D. J., Zeil, S.J., "A Formal Evaluation of Data Flow Path Selection Criteria", IEEE TSE, 15(11), Novembro, 1989, pp. 1318-1332.

[LEI05] Leitão, P. S. J., Vilela, P. R. S., Jino, Mario, "Mapping Faults to Failures in SQL Manipulation Commands", ACS/IEEE Intl. Conference on Computer Systems and Applications, Cairo, Egypt, 3-6 January, 2005.

[MAL88] Maldonado, J. C.; Chaim, M. L., Jino, M. "Seleção de Casos de Testes Baseada nos Critérios Potenciais Usos", II SBES, Canela, RS, Brasil, Outubro, 1988, pp.24-35.

[MAL91] Maldonado, J. C., "Critérios Potenciais Usos: Uma Contribuição ao Teste Estrutural de Software", Tese de Doutorado, DCA/FEEC/UNICAMP, Campinas, SP, Brasil, Julho, 1991.

[RAP82] Rapps, S., Weyuker, E.J., "Data Flow Analysis Techniques for Test Data Selection”, in Intl. Conf. on Soft. Eng., pp. 272-278, Tokio, Japan, Setembro., 1982.

[RAP85] Rapps, S., Weyuker, E.J., "Selection of Software Test Data Using Data Flow Information", IEEE TSE, SE-11(4), Abril, 1985.

[SPO00a] Spoto, E. S., Jino, M., Maldonado, J. C. "Teste Estrutural de Software: Uma abordagem para Aplicação de Banco de Dados Relacional”, SBES'00, XIV SBES, João Pessoa - PB, pp. 243-258, Outubro, 2000.

[SPO00b] Spoto, E. S., "Teste Estrutural de Programas de Aplicação de Banco de Dados Relacional", Tese de Doutorado, DCA/FEEC/UNICAMP, Campinas, SP, Brasil, Dezembro, 2000.

[WU03] Wu, X., Wang,Y., Zheng, Y. "Privacy Preserving Database Application Testing", ACM - WPES'03, October, 2003 - Washington - USA. 